\title{
Perfil Epidemiológico E Autoestima De Pacientes Queimados Em Hospital De Referência
}

\author{
Epidemiological Profile And Self-Esteem In \\ Burn Patients In A Reference Hospital
}

\author{
Sandra Renata Pinatti de Moraes' • João Fernando Marcolan²
}

\begin{abstract}
RESUMO
Objetivo: Analisar perfil sociodemográfico e autoestima em pacientes queimados de hospital referência. Método: Pesquisa quantitativa, realizada com 20 pacientes no centro de queimados de hospital público entre janeiro de 2017 a dezembro 2018. Utilizou-se questionário semiestruturado para caracterização de perfil, Escala de Autoestima de Rosenberg na alta hospitalar ou primeiro retorno após alta. Resultado: Maioria do sexo masculino (75\%), média de idade 39,49 anos, casados (65\%), com rede social comunitária $(60 \%)$, acidente doméstico em $50 \%$ dos casos, queimaduras $2^{\circ}$ grau (65\%). Média 43[34-58] dias internados, agente predominante o fogo (30\%). Melhores escores de autoestima presentes em pacientes do sexo masculino, até $4 \mathrm{I}$ anos, casado e com acesso as redes sociais. Conclusão: Ser do sexo masculino, casado e ter redes sociais teve melhores escores para autoestima em pacientes internados por queimaduras em sua maioria por acidente doméstico, com fogo, queimaduras de segundo grau em 15 a $30 \%$ de área corporal queimada.
\end{abstract}

Descritores: Unidades de queimados; cuidado de enfermagem; saúde mental; autoestima; enfermagem.

\begin{abstract}
Objective:To analyze the sociodemographic and self-esteem profile in burn patients at the hospital reference. Method: A qualitative study, with 20 patients from the burn-center in a public hospital between January 2017 to December 2018. A semi-structured questionnaire was used to characterize the sample, the Rosenberg self-esteem scale, at the moment of hospital discharge or the first control after it. Result: Most of the patients were men (75\%), 39.49 years of age (average), married (65\%), with social support (60\%), burn injuries caused by domestic accident (50\%), seconddegree burns (65\%), fire as predominant agent (30\%). The average of hospitalization was 43 days [34-58]. Men up to $4 \mathrm{l}$ years old, married and with social support presented best self-esteem scores. Conclusion: Being man, married with social support is associated with better self-esteem scores in burn patients hospitalized, mostly, with second-degree burns caused by fire flame in a domestic accident (body area affected: 15\%-30\%).

Key words: burns units; nursing care; mental health; self-esteem; nursing.
\end{abstract}




\section{INTRODUÇÃO}

A queimadura é o mais violento trauma que ocorre com a pele, acarreta problemas estéticos, psicológicos, sociais e de funcionamento físico, imediato e tardio na vida do indivíduo. É grave problema de saúde pública mundial, uma das principais causas de morbidade e mortalidade devido à complexidade do tratamento e aos poucos investimentos voltados especificamente a esta área ${ }^{(1)}$.

Segundo a Organização Mundial da Saúde (OMS) cerca de II milhões de pessoas necessitam de atendimento médico devido a lesões decorrentes das queimaduras a cada ano e 300 mil pessoas morrem por queimaduras relacionadas ao fogo, $96 \%$ em países de baixa e média renda, resultante do baixo investimento em prevenção e atendimento em centro especializado ${ }^{(2-3)}$.

A lesão por queimadura provoca trauma físico, dor intensa, internação prolongada e onerosa, no entanto, tratamento com tecnologia avançada, permite maior sobrevivência na fase aguda, mas cicatrizes e deformidades nos pacientes queimados ainda acarretam prejuízo na autoestima, ocasionando transtornos depressivos, sociais e de ajustamento, dificultando a reelaboração e reinserção de nova identidade pessoal( ${ }^{(1)}$. $\mathrm{Na}$ fase de aceitação dessa nova vida o paciente passa a viver suas maiores angústias. As cicatrizes apontam a imagem desfigurada "sentida na pele" e "observada no espelho", acompanharão o indivíduo por toda a vida e retomar o convívio em sociedade não é papel nada fácil, por isso o apoio e intervenção da equipe de saúde são primordiais ${ }^{(4)}$.

A autoestima é considerada importante indicador de saúde mental, e está relacionada a sentimentos e pensamentos que o indivíduo tem sobre o seu valor como se vê e o que pensa sob si próprio porque a aparência desagradável pode resultar em impacto negativo sobre a autoestima, sendo necessários mais estudos a respeito. A considerar que o enfermeiro é o agente que permanece ao lado do paciente durante toda internação, poderá auxiliar na busca de recursos internos para o paciente amar mais a si mesmo e realizar transição suave da fase aguda da lesão para a reabilitação e reintegração social e comunitária bem-sucedida ${ }^{(5)}$.

A nossa experiência profissional com indivíduos queimados, levou-nos a interessar acerca da autoestima e avaliar se a forma positiva ou negativa que o indivíduo faz de si mesmo tem associação com fatores relacionados à queimadura.

Há carência em nosso país de estudos a respeito de queimaduras e danos provocados à saúde emocional do indivíduo. Diante do exposto, fica clara a urgência em publicações que tracem o perfil epidemiológico e investiguem como se sente o paciente queimado de modo a entender melhor os fatores causais, distribuição e maneiras de se evitar tais acidentes, e criar estratégias para reinserir este indivíduo de modo adequado na comunidade.

\section{OBJETIVO}

Analisar o perfil sociodemográfico e autoestima em pacientes queimados de hospital escola público no Paraná.

\section{MÉTODO}

Trata-se de estudo quantitativo por amostra de conveniência, não probabilística, com pacientes internados no Centro de Tratamento a Queimados (CTQ), localizado na região norte do estado do Paraná e centro de referência a toda região. Conta com 16 leitos, sendo seis leitos de UTI e dez leitos em enfermaria.

A coleta de dados se deu de janeiro de 2017 a dezembro 2018, os dados não foram concluídos e a coleta está em andamento.

Participaram do estudo 20 pacientes com 18 anos ou mais, de ambos os sexos com queimadura de qualquer etiologia, sem problemas cognitivos e tenham permanecido pelo menos 30 dias internados.

Essa pesquisa seguiu preceitos éticos e coleta de dados ocorreu após aprovação pelo CEP da Universidade Federal de São Paulo e Universidade Estadual de Londrina sob os números: 58578416900005505 e $585784 \mid 6.9 .3001 .523$ I, respectivamente.

Depois do aceite verbal, data, horário e local para entrevista foram agendados. Previamente ao início da entrevista, os preceitos do estudo foram explicados aos pacientes eTermo de Consentimento Livre e Esclarecido (TCLE) assinado, garantido privacidade e anonimato. Entrevista ocorreu de forma individual em local privativo no ambulatório do CTQ com duração média de 40 minutos. Avaliação ocorreu na alta hospitalar com dois pacientes e os demais no primeiro retorno ambulatorial, cerca de uma semana depois. Utilizou-se questionário semiestruturado desenvolvido pelos pesquisadores para avaliar características sociodemográficas: idade, sexo, escolaridade, estado civil, ocupação, área corporal atingida pela queimadura, profundidade e extensão da queimadura, agente causador e circunstância do acidente. Aplicou-se Escala de Autoestima de Rosenberg (EAR) $)^{(6)}$, considerada confiável e ferramenta válida quantitativamente para avaliar autoestima. Avalia o sentimento positivo ou negativo do indivíduo sobre si mesmo em diferentes circunstâncias da vida. Contém 10 questões de até 30 pontos e os melhores valores são os próximos de zero(7). Possui quatro possibilidades de resposta: zero(concordo plenamente), I (concordo), 2(discordo) e 3(discordo plenamente). Durante a coleta, cartões com resposta foram expostos ou lidos ou ambos pelo entrevistador para o entrevistado apontar aquela que se assemelhava com o que sentia naquele momento.

Durante a avaliação de áreas queimadas, denominaram-se áreas expostas aquelas de difícil cobertura pela vestimenta, como: cabeça, pescoço, membros superiores e mãos e as demais como áreas não expostas.

Dados coletados foram arquivados em planilhas do Microsoft Excel 2010. Programa estatístico utilizado foi $R$ versão 3.4.2 (2017) $)^{(8)}$ e teste de aderência, Chi-quadrado, para verificar se houve associação entre resultados do questionário de Rosenberg com características sociodemográficas dos pacientes. Teste de Mann-Whitney utilizado para comparação entre escores masculino e feminino da escala.

\section{RESULTADOS}

$\mathrm{Na}$ Tabela I características sociodemográficas dos participantes.

Tabela I - Características sociodemográficas dos participantes. Londrina, Paraná. 2019. $(n=20)$.

\begin{tabular}{|c|c|c|c|}
\hline Características & & $n$ & $\%$ \\
\hline \multicolumn{4}{|l|}{ Gênero } \\
\hline & Masculino & 15 & 75 \\
\hline & Feminino & 5 & 25 \\
\hline \multicolumn{4}{|l|}{ Faixa Etária } \\
\hline & $<4 \mid$ & 11 & 55 \\
\hline
\end{tabular}




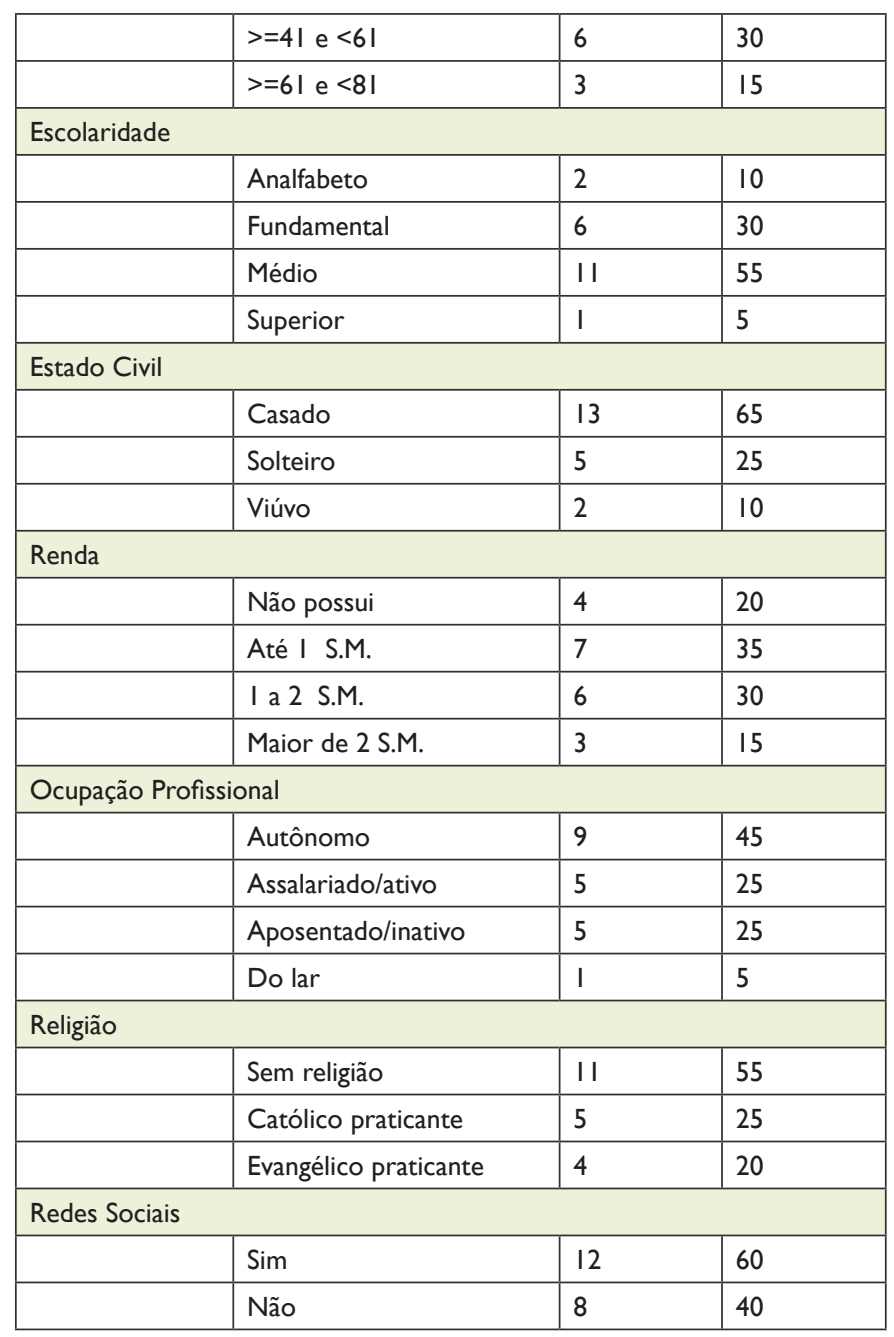

$\mathrm{Na}$ Tabela 2 dados das características da queimadura.

Tabela 2 - Características relacionadas às queimaduras. Londrina, Paraná. 2019. (n=20).

\begin{tabular}{|l|l|l|}
\hline \multicolumn{2}{|l|}{$(\mathbf{n = 6 6 3 )}$} & $\mathbf{( \% )}$ \\
\hline Idade & \multicolumn{1}{|l|}{} \\
\hline I3 a I9 & 10 & 1,5 \\
\hline 20 a 29 & 36 & 5,4 \\
\hline 30 a 39 & 29 & 4,4 \\
\hline 40 a 49 & 47 & 7,1 \\
\hline 50 a 59 & 72 & 10,9 \\
\hline 60 ou + & 469 & 70,7 \\
\hline Sexo & \multicolumn{2}{|l|}{} \\
\hline Masculino & 304 & 45,9 \\
\hline Feminino & 359 & 54,1 \\
\hline Raça/Cor & \multicolumn{2}{|l|}{} \\
\hline Branca & 202 & 30,5 \\
\hline Parda/Preta & $46 I$ & 69,5 \\
\hline Situação conjugal & \multicolumn{2}{|l|}{} \\
\hline Casado & 250 & 37,7 \\
\hline Solteiro & 263 & 39,7 \\
\hline $\begin{array}{l}\text { Desquitado/separado/divor- } \\
\text { ciado }\end{array}$ & 15 & 2,3 \\
\hline Viúvo & 92 & 13,9 \\
\hline Amasiado & 20 & 3 \\
\hline Não informado & 23 & 3,5 \\
\hline
\end{tabular}

\begin{tabular}{|l|l|l|}
\hline \multicolumn{3}{|l|}{ Descrição de presença de fatores de risco } \\
\hline Não & 23 & 3,5 \\
\hline Sim & 451 & 68 \\
\hline Não informado & 189 & 28,5 \\
\hline Descrição de medicamentos em uso contínuo \\
\hline Não & 72 & 10,9 \\
\hline Sim & 443 & 66,8 \\
\hline Não informado & 148 & 22,3 \\
\hline Óbito & 618 & 93,2 \\
\hline Não & 45 & 6,8 \\
\hline Sim & *Período médio de internação: 43[34-58] dias.
\end{tabular}

A maioria dos casos de queimaduras (80\%) resultou em longo período de internação.

Tabela 3 com resultados para autoestima e características sociodemográficas.

Tabela 3 - Dados da Escala de Autoestima de Rosenberg e características sociodemográficas. Londrina- Paraná. 2019. $(n=20)$.

\begin{tabular}{|c|c|c|c|c|}
\hline Variável & AE alta & AE média & AE baixa & p-valor \\
\hline \multicolumn{4}{|l|}{ Sexo } & $0,60 \mathrm{NS}$ \\
\hline Masculino & $8(40 \%)$ & $7(35 \%)$ & $0(0 \%)$ & \\
\hline Feminino & $4(20 \%)$ & $\mathrm{I}(5 \%)$ & $0(0 \%)$ & \\
\hline \multicolumn{4}{|l|}{ Idade } & $0,40 \mathrm{NS}$ \\
\hline$<41$ & $5(25 \%)$ & $6(30 \%)$ & $0(0 \%)$ & \\
\hline$>=4 \mid$ e $<6 \mid$ & $4(20 \%)$ & $2(10 \%)$ & $0(0 \%)$ & \\
\hline$>=61$ e $<8 \mid$ & $3(15 \%)$ & $0(0 \%)$ & $0(0 \%)$ & \\
\hline \multicolumn{4}{|l|}{ Estado Civil } & 0,08 \\
\hline Casado & $9(45 \%)$ & $4(20 \%)$ & $0(0 \%)$ & \\
\hline Solteiro & $4(20 \%)$ & $4(20 \%)$ & $0(0 \%)$ & \\
\hline Viúvo & $2(10 \%)$ & $0(0 \%)$ & $0(0 \%)$ & \\
\hline \multicolumn{4}{|l|}{ Renda } & $0,58 \mathrm{NS}$ \\
\hline Não possui & $2(10 \%)$ & $2(10 \%)$ & $0(0 \%)$ & \\
\hline Até Ism & $3(15 \%)$ & $4(20 \%)$ & $0(0 \%)$ & \\
\hline De I a $2 \mathrm{sm}$ & $4(20 \%)$ & $2(10 \%)$ & $0(0 \%)$ & \\
\hline $\begin{array}{l}\text { Maior de } \\
2 \mathrm{sm}\end{array}$ & $3(15 \%)$ & $0(0 \%)$ & $0(0 \%)$ & \\
\hline \multicolumn{4}{|l|}{ Escolaridade } & $0,57 \mathrm{NS}$ \\
\hline Analfabeto & $2(10 \%)$ & $0(0 \%)$ & $0(0 \%)$ & \\
\hline Fundamental & $3(15 \%)$ & $3(15 \%)$ & $0(0 \%)$ & \\
\hline Médio & $6(30 \%)$ & $5(25 \%)$ & $0(0 \%)$ & \\
\hline Superior & $\mathrm{I}(5 \%)$ & $0(0 \%)$ & $0(0 \%)$ & \\
\hline \multicolumn{4}{|l|}{ Redes Sociais } & $0,35 \mathrm{NS}$ \\
\hline Possui & $9(45 \%)$ & $3(15 \%)$ & $0(0 \%)$ & \\
\hline Não Possui & $3(15 \%)$ & $5(25 \%)$ & $0(0 \%)$ & \\
\hline \multicolumn{4}{|c|}{ Grau da queimadura } & $0,46 \mathrm{NS}$ \\
\hline $2^{\circ}$ grau & $7(35 \%)$ & $6(30 \%)$ & $0(0 \%)$ & \\
\hline $3^{\circ}$ grau & $3(15 \%)$ & $2(10 \%)$ & $0(0 \%)$ & \\
\hline $\begin{array}{l}\text { Misto em } 2^{\circ} \\
\text { e } 3^{\circ} \mathrm{grau}\end{array}$ & $2(10 \%)$ & $0(0 \%)$ & $0(0 \%)$ & \\
\hline
\end{tabular}

NS- não significativo

A média global para autoestima foi de 9,05 pontos. Pacientes do sexo masculino, idade inferior a $4 \mathrm{I}$ anos, casados, com renda de até dois salários mínimos, apresentaram melhor autoestima que mulheres. Pacientes com queimaduras de segundo grau com melhor nível de escolaridade tinham mais 
acesso as redes sociais e melhor autoestima.

$\mathrm{Na}$ Tabela 4 dados sobre autoestima em relação à área exposta queimada por sexo.

Tabela 4 - Resultados da Escala de autoestima de Rosenberg em relação à área exposta queimada por sexo. LondrinaParaná. $(\mathrm{N}=20)$.

\begin{tabular}{|l|l|l|l|l|}
\hline \multicolumn{1}{|l|}{ Baixa AE } & Média AE & Alta AE & p-valor \\
\hline Masculino & $0(0 \%)$ & $4(26,67 \%)$ & $7(46,66 \%)$ & \\
\hline$<=15 \%$ & $0(0 \%)$ & $3(20 \%)$ & $\mathrm{I}(6,67 \%)$ & \\
\hline $\begin{array}{l}>15 \% \text { e } \\
<=30 \%\end{array}$ & 0,29 & \\
\hline $\begin{array}{l}>30 \% \text { e }<= \\
45 \%\end{array}$ & $0(0 \%)$ & $0(0 \%)$ & $0(0 \%)$ & \\
\hline$>45 \%$ & $0(0 \%)$ & $0(0 \%)$ & $0(0 \%)$ & \\
\hline \begin{tabular}{l} 
Feminino \\
\hline$<=15 \%$
\end{tabular} & $0(0 \%)$ & $\mathrm{I}(20 \%)$ & $2(40 \%)$ & \\
\hline $\begin{array}{l}>15 \% \text { e }<= \\
30 \%\end{array}$ & $0(0 \%)$ & $0(0 \%)$ & $2(40 \%)$ & \\
\hline $\begin{array}{l}>30 \% \text { e }<= \\
45 \%\end{array}$ & $0(0 \%)$ & $0(0 \%)$ & $0(0 \%)$ & \\
\hline$>45 \%$ & $0(0 \%)$ & $0(0 \%)$ & $0(0 \%)$ & \\
\hline
\end{tabular}

$\mathrm{Na}$ Tabela 5 estão dados sobre autoestima e área não exposta e sexo.

Tabela 5 - Resultados da Escala de autoestima de Rosenberg em relação à área não exposta queimada por sexo. LondrinaParaná. $(\mathrm{N}=20)$.

\begin{tabular}{|l|l|l|l|l|}
\hline \multicolumn{1}{|c|}{ Baixa AE } & \multicolumn{1}{|c|}{ Média AE } & Alta AE & p-valor \\
\hline Masculino & \multicolumn{1}{|c|}{} & 0,45 \\
\hline$<=15 \%$ & $0(0 \%)$ & $6(40 \%)$ & $6(40 \%)$ & \\
\hline $\begin{array}{l}>15 \% \text { e } \\
<=30 \%\end{array}$ & $0(0 \%)$ & $\mathrm{I}(6,6 \%)$ & $\mathrm{I}(6,67 \%)$ & \\
\hline $\begin{array}{l}>30 \% \text { e }<= \\
45 \%\end{array}$ & $0(0 \%)$ & $0(0 \%)$ & $\mathrm{I}(6,67 \%)$ & \\
\hline$>45 \%$ & $0(0 \%)$ & $0(0 \%)$ & $0(0 \%)$ & \\
\hline \begin{tabular}{l} 
Feminino \\
\hline$<=15 \%$
\end{tabular} & $0(0 \%)$ & $\mathrm{I}(20 \%)$ & $2(40 \%)$ & \\
\hline $\begin{array}{l}>15 \% \text { e }<= \\
30 \%\end{array}$ & $0(0 \%)$ & $0(0 \%)$ & $2(40 \%)$ & \\
\hline $\begin{array}{l}>30 \% \text { e }<= \\
45 \%\end{array}$ & $0(0 \%)$ & $0(0 \%)$ & $0(0 \%)$ & \\
\hline$>45 \%$ & $0(0 \%)$ & $0(0 \%)$ & $0(0 \%)$ & \\
\hline
\end{tabular}

Pelo teste de aderência ao chi-quadrado não encontrada associação significativa entre autoestima e área corporal exposta e não exposta queimada e sexo.

Indivíduos do sexo masculino quando investigados quanto à exposição ou não da área queimada, apresentaram autoestima superior ao sexo feminino. Comparada pontuação de autoestima entre homens e mulheres pelo teste de MannWhitney, sexo masculino obteve pontuação melhor que feminino $(p=0,04)$, devido a proporção maior nesse grupo.

Testes estatísticos de aderência ao chi-quadrado não encontraram associação significativa entre autoestima e área corporal exposta e não exposta por queimadura e sexo. Indivíduos do sexo feminino investigados quanto à área exposta e não exposta apontaram autoestima superior quando área queimada não ficou aparente, sexo masculino não apresentou diferença entre área exposta e não exposta queimada e autoestima.

\section{DISCUSSÃO}

Observamos incidência de queimaduras maior no sexo masculino, provavelmente pela cultura brasileira, onde homem precisa ser forte, racional e assertivo, por ter menos cuidado no desempenho de atividades, sofrer maior risco de acidentes corroborando com literatura nacional ${ }^{(4,9)}$ e internacional ${ }^{(10-11)}$, apontaram que homens estão mais expostos a atividades laborais perigosas comuns na prática masculina como manuseio de eletricidade e produtos químicos.

Observamos quanto à idade, faixa etária produtiva, economicamente ativa foi a mais atingida, como outros estudos epidemiológicos ${ }^{(9,11-12)}$. Queimadura ocasiona problemas de ordem econômica, social, familiar, alteração na aparência física e capacidade funcional, indivíduo muitas vezes desenvolve problemas psicológicos como baixa autoestima ${ }^{(11)}$.

Pela escolaridade, notamos baixo grau de instrução dos entrevistados, oscilando entre fundamental e médio; embora não encontramos estudos voltados à escolaridade do paciente queimado, inferimos que tal agravo é mais comum em países de baixa renda e relacionado a pouco investimento em prevenção. Encontramos dados semelhantes em indianos, maioria da amostra estudou até nível médio(II).

Quanto status de trabalho, maioria assalariada ou autônoma, baixa remuneração associada à baixa escolaridade, como outros estudos nacionais ${ }^{(4,9)}$. No Brasil, queimaduras estão associadas à baixa renda, pobreza, baixo nível educacional; queimadura e status econômico estão interligados, segundo $\mathrm{OMS}^{(13)}$.

Período de internação dos participantes foi longo relacionado à extensão e gravidade da queimadura. Período de internação interfere em questões econômicas devido ausência ao trabalho, consequente falta de recursos para provisão da família, distanciamento do ente querido devido longa internação. Estudo semelhante avaliando qualidade de vida no queimado também encontrou período de permanência similar ${ }^{(9)}$. Estudo avaliou a saúde após queimadura, obteve período de internação inferior a 30 dias $^{(4)}$.

Ambiente doméstico foi onde ocorreu maioria dos acidentes, corroborando com estudo em metrópole urbana da Índia ${ }^{(11)}$ e estudos brasileiros ${ }^{(14-15)}$ devido à falta de atenção dos indivíduos ao realizar atividades domésticas (cozinhar, manipular produtos de limpeza) e trabalho externo com atividades insalubres com líquidos inflamáveis e combustíveis ${ }^{(16)}$. A maior parte dos acidentes ocorridos no ambiente doméstico ou de trabalho poderia ser evitada com implementação de medidas de prevenção.

Nosso estudo aponta quanto à etiologia, predomínio da queimadura térmica, agente causal mais comum o fogo, seguido do agente elétrico arco voltaico. Provavelmente, esse tipo de acidente devido à predominância do gênero masculino e tipo de trabalho.A sequela pode provocar comprometimento físico e psicológico com interferência na autoestima.

Estudo realizado na Ásia para avaliar autoestima de indivíduos gravemente queimados, ${ }^{(12)}$ não encontrou diferença significativa entre gêneros e autoestima e variáveis como idade, estado civil, escolaridade. Em nosso estudo, não encontramos diferença quando avaliada variáveis sociodemográficas, pela amostra pequena, no entanto, quando comparamos autoestima entre os sexos, homens obtiveram pontuação melhor, porém tiveram quantidade maior.

Predominância da queimadura de segundo grau, superfície corpórea se concentrou de 15 a $30 \%$, semelhantes aos achados em outros estudos(4,II). 
Cicatrizes resultantes das queimaduras são sequelas mais difíceis de serem tratadas porque podem provocar danos à saúde mental e impactar seu comportamento social. Estudo indiano apontou para menor profundidade da queimadura melhor autoestima, sem apontar diferença estatística significativa( ${ }^{(I)}$. Verificamos que profundidade das queimaduras não apontou significância, mas com melhor pontuação em superfície de menor profundidade.

Autoestima é importante indicador de saúde mental, está relacionada a sentimentos e pensamentos que o indivíduo tem sobre seu valor, como se vê, o que pensa sobre si mesmo e a refletir de modo positivo ou negativo em sua vida pessoal ${ }^{(6)}$.

Avaliação da autoestima em pacientes queimados vem despertando interesse dos pesquisadores ${ }^{(17,18)}$ devido a alteração na aparência, principalmente das cicatrizes visíveis, prejudicarem o bem estar emocional acarretando isolamento social.

Paciente queimado apresenta sequelas físicas e na imagem corporal, podendo influenciar sua autoestima. Com o passar do tempo e das necessidades de vida, desenvolve meios de adaptação à nova condição para levar a vida de modo real(17).

Estudos $^{(19-20)}$ utilizam diferentes pontuações para Escala de Autoestima de Rosenberg (EAR) (7), dificultando comparação com outros trabalhos. Em nosso estudo, média de autoestima foi satisfatória, como encontrado em estudo com similar classificação abordando jovens queimados que foi de 8,4 I pontos $^{(7)}$. Diferenças nos escores de autoestima quanto variáveis sociodemográficas (sexo, idade, escolaridade, renda) não foram encontradas, corroborando com estudo asiático(12).

Sexo feminino apresentou menor autoestima, associamos tal fato à excessiva cobrança à imagem corporal e padrões de beleza pré-estabelecido em nosso país ${ }^{(18)}$. Paciente encontra dificuldades e experiência desafios complexos, físicos e psicológicos, podendo sentir-se menos atraente pelas cicatrizes, dificultando sua vida de relação. Engajar família é salutar no processo de recuperação.

Estudo tem por limitações ser menos generalizável pela amostra pequena, clientela com problema específico e de único centro.

\section{CONCLUSÃO}

Gênero masculino, jovem, com baixa escolaridade, queimadura de segundo grau, pelo fogo foram predominantes. Lesões acarretaram mudanças no indivíduo e cicatrizes físicas podem resultar em problemas emocionais. Autoestima foi melhor no sexo masculino.

Pesquisas devem se concentrar em estudos de longo prazo em diferentes centros para clarificar os fatores emocionais relacionados à autoestima do indivíduo com queimadura e contribuir no planejamento de intervenções que visem reduzir sofrimento e da aceitação do indivíduo em seu cotidiano para a vida saudável.

\section{REFERÊNCIAS}

1. Sideli L, Prestifilippo A, Di Benedetto B, Farrauto R, Grassia R, Mulè A, et al. Quality of life, body image, and psychiatric complications in patients with a burn trauma: preliminary study of the Italian version of the burn specific health-scale brief.Ann Burns fire disasters [Internet]. 2010 [acesso em 07 jan 2019]; 23(4): I7I6. Disponível em: https://www.ncbi.nlm.nih.gov/pmc/ articles/PMC3 I88270/
2. World Health Organization. A WHO plan for burn prevention and care. Geneva: World Health Organization; 2008 [acesso em 20 fev 2019]. Disponível em: https://apps.who.int/iris/bitstream/ handle/l0665/97852/978924/596299_eng. pdf? sequence $=\mid$ \&isAllowed $=y$

3. Peck MD. Epidemiology of burns throughout the world. Part I. Distribution and risk factors. Burns [Internet]. 20I I [acesso em I0 jan 20I9]; 37(7):I087100. Disponível em: https://www.ncbi.nlm.nih.gov/ pubmed/2I802856.

4. Ricci H,Gonçalves N, Gallan MC, Ciol MA, Dantas RAS, Rossi LA. Assessment of the health status in Brazilian burns victims five to seven months after hospital discharge. Burns [Internet]. 2014 [acesso em 2019 jan 10];40(4):616-23. Disponível em: https://www.ncbi. nlm.nih.gov/pubmed/24290853

5. Branden N. O poder da autoestima. $6^{a}$ ed. São Paulo: Saraiva; 2005.

6. Dini DM, Quaresma MR, Ferreira LM. Adaptação cultural e validação da versão brasileira da escala de autoestima de Rosenberg. Rev Soc Bras Cir Plast [Internet]. 2004 [acesso em 20 jan 2019]; I9(I):4I52. Disponível em: http://www.rbcp.org.br/details/322/ adaptacao-cultural-e-validacao-da-versao-brasileirada-escala-de-auto-estima-de-rosenberg.

7. Teixeira NJ, Carvalho VF, Llonch Sabatés A. A quantitative cross-sectional of depression and selfesteem in teenage and young adult burn victims in rehabilitation. Ostomy Wound Manege. 2013 [acesso em 20 jan 2019]; 59(9):22-9. Disponível em: https:// www.ncbi.nlm.nih.gov/pubmed/24018389

8. R Core Team.A language and environment for statistical computing. (Version 3.4. 2)[Computer software]. Vienna,Austria: R Foundation for Statistical Computing; 2017. Disponível em: https://www.r-project.org/.

9. Amaral Zorita L, Blanes L, Francescato Veiga D, Silva Augusto F, Masako Ferreira L. Health-related quality of life and self-esteem among burn patients. Wounds [Internet]. 2016 [acesso em 18 abr 2019]; 28(I):2734. Disponível em: https://www.woundsresearch.com/ article/health-related-quality-life-and-self-esteemamong-burn-patients.

10. Thakrar S, Hunter TA, Medved MI, Hiebert-Murphy D, Jens Brockmeier J, Sareen J, et al. Men, fire, and burns: stories of fighting, healing, and emotions. Burns [Internet]. 20I5. [acesso em I8 abr I0]; 4I(8):I66473. Disponível em: https://www.sciencedirect.com/ science/article/pii/S03054I79I500I62X?via\%3Dihub

11. Jain M, Khadilkar N, Souza A. Burn-related factors affecting anxiety, depression and self-esteem in burn patients: an exploratory study.Ann Burns Fire Disaters [Internet]. 2017 [acesso em 18 mar 2019]; 30(I):304. Disponível em: https://www.ncbi.nlm.nih.gov/pmc/ articles/PMC5446905/

12. Zaidi SMIH,Yaqoob N, Noreen S. Self-esteem in severely burned adults.J Pak Med Assoc [Internet]. 2017 [acesso em I8 jan 10]; 67(12):1914-16. Disponível em: https:// www.researchgate.net/publication/32 I 314500_Selfesteem_in_severely_burned_adults

13. World Health Organization. Burns. Geneva: WHO; 2018 [acesso em 20 fev 2019]. Disponível em: https:// 
www.who.int/en/news-room/fact-sheets/detail/burns

14. Montes SF, Barbosa MH, Souza Neto AL. Aspectos clínicos e epidemiológicos de pacientes queimados internados em um hospital de ensino. Rev Esc EnfermUSP [Internet]. 20I I [acesso em 20 fev 20I9]; 45(2):369-73. Disponível em: http://www.scielo.br/pdf/ reeusp/v45n2/v45n2a09.pdf.

15. Gonçalves N, Rodrigues RB, Oliveira HC, Novaes FN, Rossi LA, Rodrigues RCM. Cultural adaptation of the 5-D itch scale and its reliability for Brazilian burn survivors. Burns [Internet]. 2018 [acesso em 13 fev 2019]; 45(3):717-24. Disponível em: https://www.sciencedirect.com/science/article/pii/ S03054 I79/8308404?via\%3Dihub

16. Vendrúsculo TM, Baliero CRB, Echevarria-Guanilo ME, Júnior JAF, Rossi LA. Queimaduras em ambientes domésticos: características e circunstâncias do acidente. Rev Latino-Am Enferm [Internet]. 2010 [acesso 20 fev 2019]; 18(3):444-5I. Disponível em: http://www.scielo.br/pdf/rlae/v18n3/pt_2l.pdf

17. Laporte GA, Leonardi DF. Transtorno de estresse pós-traumático em pacientes com sequelas de queimaduras. Rev Bras Queimaduras [Internet]. 2010 [acesso em 20 abr 2019]; 9(3):105-14. Disponível em: http://webcache.googleusercontent. com/search? q=cache: $\mathbf{Z} Z \mathrm{~s} 3 \mathrm{VWKXCNcJ:www.}$
rbqueimaduras.com.br/details/44/pt-BR/transtornode-estresse-pos-traumatico-em-pacientescom-sequelas-de-queimaduras $+\& c d=|\& h|=p t-$ $\mathrm{BR} \& \mathrm{ct}=\mathrm{clnk} \& \mathrm{gl}=\mathrm{br}$

18. Silva MF, Silva MJP. A auto-estima de pacientes ambulatoriais com queimaduras. Rev Baiana Enferm [Internet]. 2002 [acesso 20 jan 2019]; 17(3):75-84. Disponível em: https://portalseer.ufba.br/index.php/ enfermagem/article/view/3863/2829

19. Jang $M H$, Park J, Chong MK, Sok SR. Factors influencing resilience of burns patients in South Korea. J Nurs Scholarsh [Internet]. 2017 [acesso em 20 jan 2019]; 49(5):478-86. Disponível em: https://sigmapubs. onlinelibrary.wiley.com/doi/full/I0. I I I I/jnu. I23 I I.

20. Hoogewerf CJ, Van Baar ME, Middelkoop E, Van Loey NE. Impact of facial burns: relationship between depressive symptoms, self-esteem and scar severity. Gen Hosp Psychiatry [Internet]. 2014 [acesso em 20 jan 20l9]; 36 (20l4):27I-6. Disponível em: https://www.sciencedirect.com/science/article/pii/ Sol638343/3003666?via\%3Dihub.
Recebido: 2019-07-31

Aceito: 2019-10-18 